\title{
On unit group of finite semisimple group algebras of non-metabelian groups of order 108
}

\author{
Gaurav Mittal, Rajendra. K. Sharma
}

\begin{abstract}
In this paper, we characterize the unit groups of semisimple group algebras $\mathbb{F}_{q} G$ of non-metabelian groups of order 108, where $F_{q}$ is a field with $q=p^{k}$ elements for some prime $p>3$ and positive integer $k$. Upto isomorphism, there are 45 groups of order 108 but only 4 of them are non-metabelian. We consider all the non-metabelian groups of order 108 and find the Wedderburn decomposition of their semisimple group algebras. And as a by-product obtain the unit groups.
\end{abstract}

2010 MSC: $16 \mathrm{U} 60,20 \mathrm{C} 05$

Keywords: Unit group, Finite field, Wedderburn decomposition

\section{Introduction}

Let $\mathbb{F}_{q}$ denote a finite field with $q=p^{k}$ elements for odd prime $p>3, G$ be a finite group and $\mathbb{F}_{q} G$ be the group algebra. The study of the unit groups of group algebras is a classical problem and has applications in cryptography [4] as well as in coding theory [5] etc. For the exploration of Lie properties of group algebras and isomorphism problems, units are very useful see, e.g. [1]. We refer to [11] for elementary definitions and results about the group algebras and $[2,14]$ for the abelian group algebras and their units. Recall that a group $G$ is metabelian if there is a normal subgroup $N$ of $G$ such that both $N$ and $G / N$ are abelian. The unit groups of the finite semisimple group algebras of metabelian groups have been well studied.

In this paper, we are concerned about the unit groups of the group algebras of non-metabelian groups. Let us first mention the available literature in this direction. From [13], we know that all the groups up to order 23 are metabelian. The only non-metabelian groups of order 24 are $S_{4}$ and $S L(2,3)$ and the unit group of their group algebras have been discussed in [7, 9]. Further, from [13], we also

Gaurav Mittal (Corresponding Author); Department of Mathematics, Indian Institute of Technology Roorkee, Roorkee, India (email: gmittal@ma.iitr.ac.in).

R. K. Sharma; Department of Mathematics, Indian Institute of Technology Delhi, New Delhi, India (email: rksharma@maths.iitd.ac.in). 
deduce that there are non-metabelian groups of order of 48,54,60,72,108 etc. The unit groups of the group algebras of non-metabelian groups up to order 72 have been discussed in [10, 12]. The unit group of the semisimple group algebra of the non-metabelian group $S L(2,5)$ has been discussed in [15].

The main motive of this paper is to characterize the unit groups of $\mathbb{F}_{q} G$, where $G$ represents a non-metabelian group of order 108. It can be verified that, upto isomorphism, there are 45 groups of order 108 and only 4 of them are non-metabelian. We deduce the Wedderburn decomposition of group algebras of all the 4 non-metabelian groups and then characterize the respective unit groups. The rest of the paper is organized in following manner: We recall all the basic definitions and results to be needed in our work in Section 2. Our main results on the characterization of the unit groups are presented in third section and the last section includes some discussion.

\section{Preliminaries}

Let $e$ denote the exponent of $G, \zeta$ a primitive $e^{t h}$ root of unity. On the lines of [3], we define

$$
I_{\mathbb{F}}=\left\{n \mid \zeta \mapsto \zeta^{n} \text { is an automorphism of } \mathbb{F}(\zeta) \text { over } \mathbb{F}\right\}
$$

where $\mathbb{F}$ is an arbitrary finite field. Since, the Galois group $\operatorname{Gal}(\mathbb{F}(\zeta), \mathbb{F})$ is a cyclic group, for any $\tau \in$ $\operatorname{Gal}(\mathbb{F}(\zeta), \mathbb{F})$, there exists a positive integer $s$ which is invertible modulo $e$ such that $\tau(\zeta)=\zeta^{s}$. In other words, $I_{\mathbb{F}}$ is a subgroup of the multiplicative group $\mathbb{Z}_{e}^{*}$ (group of integers which are invertible with respect to multiplication modulo $e$ ). For any $p$-regular element $g \in G$, i.e. an element whose order is not divisible by $p$, let the sum of all conjugates of $g$ be denoted by $\gamma_{g}$, and the cyclotomic $\mathbb{F}$-class of $\gamma_{g}$ be denoted by $S\left(\gamma_{g}\right)=\left\{\gamma_{g^{n}} \mid n \in I_{\mathbb{F}}\right\}$. The cardinality of $S\left(\gamma_{g}\right)$ and the number of cyclotomic $\mathbb{F}$-classes will be incorporated later on for the characterization of the unit groups.

Next, we recall two important results from [3]. First one relates the number of cyclotomic $\mathbb{F}$-classes with the number of simple components of $\mathbb{F} G / J(\mathbb{F} G)$. Here $J(\mathbb{F} G)$ denotes the Jacobson radical of $\mathbb{F} G$. Second one is about the cardinality of any cyclotomic $\mathbb{F}$-class in $G$.

Theorem 2.1. The number of simple components of $\mathbb{F} G / J(\mathbb{F} G)$ and the number of cyclotomic $\mathbb{F}$-classes in $G$ are equal.

Theorem 2.2. Let $\zeta$ be defined as above and $j$ be the number of cyclotomic $\mathbb{F}$-classes in $G$. If $K_{i}, 1 \leq$ $i \leq j$, are the simple components of center of $\mathbb{F} G / J(\mathbb{F} G)$ and $S_{i}, 1 \leq i \leq j$, are the cyclotomic $\mathbb{F}$-classes in $G$, then $\left|S_{i}\right|=\left[K_{i}: \mathbb{F}\right]$ for each $i$ after suitable ordering of the indices if required.

To determine the structure of unit group $U(\mathbb{F} G)$, we need to determine the Wedderburn decomposition of the group algebra $\mathbb{F} G$. In other words, we want to determine the simple components of $\mathbb{F} G$. Based on the existing literature, we can always claim that $\mathbb{F}$ is one of the simple component of decomposition of $\mathbb{F} G / J(\mathbb{F} G)$. The simple proof is given here for the completeness.

Lemma 2.3. Let $A_{1}$ and $A_{2}$ denote the finite dimensional algebras over $\mathbb{F}$. Further, let $A_{2}$ be semisimple and $g$ be an onto homomorphism between $A_{1}$ and $A_{2}$, then we must have $A_{1} / J\left(A_{1}\right) \cong A_{3}+A_{2}$, where $A_{3}$ is some semisimple $\mathbb{F}$-algebra.

Proof. From [6], we have $J\left(A_{1}\right) \subseteq \operatorname{Ker}(g)$. This means there exists $\mathbb{F}$-algebra homomorphism $g_{1}$ from $A_{1} / J\left(A_{1}\right)$ to $A_{2}$ which is also onto. In other words, we have

$$
g_{1}: A_{1} / J\left(A_{1}\right) \longmapsto A_{2} \text { defined by } g_{1}\left(a+J\left(A_{1}\right)\right)=g(a), \quad a \in A_{1} .
$$

As $A_{1} / J\left(A_{1}\right)$ is semisimple, there exists an ideal $I$ of $A_{1} / J\left(A_{1}\right)$ such that

$$
A_{1} / J\left(A_{1}\right)=\operatorname{ker}\left(g_{1}\right) \oplus I
$$


Our claim is that $I \cong A_{2}$. For this to prove, note that any element $a \in A_{1} / J\left(A_{1}\right)$ can be uniquely written as $a=a_{1}+a_{2}$ where $a_{1} \in \operatorname{ker}\left(g_{1}\right), a_{2} \in I$. So, define

$$
g_{2}: A_{1} / J\left(A_{1}\right) \mapsto \operatorname{ker}\left(g_{1}\right) \oplus A_{2} \text { by } g_{2}(a)=\left(a_{1}, g_{1}\left(a_{2}\right)\right)
$$

Since, $\operatorname{ker}\left(g_{1}\right)$ is a semisimple algebra over $\mathbb{F}$ and $A_{2}$ is an isomorphic $\mathbb{F}$-algebra, claim and the result holds.

Above lemma concludes that $\mathbb{F}$ is one of the simple components of $\mathbb{F} G$ provided $J(\mathbb{F} G)=0$. Now, we recall a result which characterize the set $I_{\mathbb{F}}$ defined in the beginning of this section. For proof, see Theorem 2.21 in [8].

Theorem 2.4. Let $\mathbb{F}$ be a finite field with prime power order $q$. If e is such that $\operatorname{gcd}(e, q)=1, \zeta$ is the primitive $e^{\text {th }}$ root of unity and $|q|$ is the order of $q$ modulo $e$, then modulo $e$, we have $I_{\mathbb{F}}=$ $\left\{1, q, q^{2}, \ldots, q^{|q|-1}\right\}$.

Next result is Proposition 3.6.11 from [11] and is useful for the determination of commutative simple components of the group algebra $\mathbb{F}_{q} G$.

Theorem 2.5. If $R G$ is a semisimple group algebra, then $R G \cong R\left(G / G^{\prime}\right) \oplus \Delta\left(G, G^{\prime}\right)$, where $G^{\prime}$ is the commutator subgroup of $G, R\left(G / G^{\prime}\right)$ is the sum of all commutative simple components of $R G$, and $\Delta\left(G, G^{\prime}\right)$ is the sum of all others.

We end this section by recalling a Proposition 3.6.7 from [11] which is a generalized version of the last result.

Theorem 2.6. Let $R G$ be a semisimple group algebra and $H$ be a normal subgroup of $G$. Then $R G \cong$ $R(G / H) \oplus \Delta(G, H)$, where $\Delta(G, H)$ is a left ideal of $R G$ generated by the set $\{h-1: h \in H\}$.

\section{Unit group of $\mathbb{F}_{q} G$ where $G$ is a non-metabelian group of order 108}

The main objective of this section is to characterize the unit group of $\mathbb{F}_{q} G$ where $G$ is a nonmetabelian group of order 108. Upto isomorphism, there are 4 non-metabelian groups of order 108 namely: (1) $G_{1}=\left(\left(C_{3} \times C_{3}\right) \rtimes C_{3}\right) \rtimes C_{4}$.

(2) $G_{2}=\left(\left(C_{3} \times C_{3}\right) \rtimes C_{3}\right) \rtimes C_{4}$.

$$
\text { (3) } G_{3}=\left(\left(C_{3} \times C_{3}\right) \rtimes C_{3}\right) \rtimes\left(C_{2} \times C_{2}\right) . \quad(4) G_{4}=C_{2} \times\left(\left(\left(C_{3} \times C_{3}\right) \rtimes C_{3}\right) \rtimes C_{2}\right) .
$$

Here $G_{1}$ and $G_{2}$ are two non-isomorphic groups formed by the semi-direct product of $\left(C_{3} \times C_{3}\right) \rtimes C_{3}$ and $C_{4}$ which will be clear once we discuss the presentation of these groups. We consider each of these 4 groups one by one and discuss the unit groups of their respective group algebras along with the Wedderburn decompositions in subsequent subsections. Throughout this paper, we use the notation $[x, y]=x^{-1} y^{-1} x y$.

\subsection{The group $G_{1}=\left(\left(C_{3} \times C_{3}\right) \rtimes C_{3}\right) \rtimes C_{4}$}

Group $G_{1}$ has the following presentation:

$$
\begin{gathered}
G_{1}=\langle x, y, z, w, t| x^{2} y^{-1},[y, x],[z, x] z^{-1},[w, x] w^{-1},[t, x], y^{2},[z, y],[w, y], \\
\left.[t, y], z^{3},[w, z] t^{-1},[t, z], w^{3},[t, w], t^{3}\right\rangle .
\end{gathered}
$$

Also $G_{1}$ has 20 conjugacy classes as shown in the table below. 


\begin{tabular}{|c|c|c|c|c|c|c|c|c|c|c|c|c|c|c|c|c|c|c|c|}
\hline $\mathrm{r}$ & $e$ & $y$ & & $w$ & $t x$ & $x y$ & $x t$ & $y z$ & $y w$ & $y t$ & $z w$ & $t^{2}$ & xyt & $x t^{2}$ & $y z w$ & $y t^{2}$ & $z^{2} w$ & $x y t^{2}$ & $y z^{2} w$ \\
\hline $\mathrm{S}$ & \begin{tabular}{l|l}
1 & 9
\end{tabular} & \begin{tabular}{|l|l|}
9 & 1 \\
\end{tabular} & 6 & 6 & 1 & 9 & 9 & 6 & 6 & 1 & 6 & 1 & 9 & 9 & 0 & 1 & 6 & 9 & 6 \\
\hline o & \begin{tabular}{l|l}
1 & 4 \\
\end{tabular} & \begin{tabular}{|l|l|}
4 & 2 \\
\end{tabular} & 3 & 3 & 3 & 4 & 12 & 6 & 6 & 6 & 3 & 3 & 12 & 12 & 6 & 6 & 3 & 12 & 6 \\
\hline
\end{tabular}

where $\mathrm{r}, \mathrm{s}$ and o denote the representative of conjugacy class, size and order of the representative of the conjugacy class, respectively. From the above discussion, it is clear that exponent of $G_{1}$ is 12 . Also $G_{1}^{\prime} \cong\left(C_{3} \times C_{3}\right) \rtimes C_{3}$ with $G_{1} / G_{1}^{\prime} \cong C_{4}$. Next, we discuss the unit group of the group algebra $\mathbb{F}_{q} G_{1}$ when $p>3$.

Theorem 3.1. The unit group of $\mathbb{F}_{q} G_{1}$, for $q=p^{k}, p>3$ where $\mathbb{F}_{q}$ is a finite field having $q=p^{k}$ elements is as follows:

1. for any $p$ and $k$ even or $p^{k} \equiv 1 \bmod 12$ with $k$ odd, we have

$$
U\left(\mathbb{F}_{q} G_{1}\right) \cong\left(\mathbb{F}_{q}^{*}\right)^{4} \oplus G L_{2}\left(\mathbb{F}_{q}\right)^{8} \oplus G L_{3}\left(\mathbb{F}_{q}\right)^{8} .
$$

2. for $p^{k} \equiv 5 \bmod 12$ with $k$ odd, we have

$$
U\left(\mathbb{F}_{q} G_{1}\right) \cong\left(\mathbb{F}_{q}^{*}\right)^{4} \oplus G L_{2}\left(\mathbb{F}_{q}\right)^{8} \oplus G L_{3}\left(\mathbb{F}_{q^{2}}\right)^{4}
$$

3. for $p^{k} \equiv 7 \bmod 12$ with $k$ odd, we have

$$
U\left(\mathbb{F}_{q} G_{1}\right) \cong\left(\mathbb{F}_{q}^{*}\right)^{2} \oplus \mathbb{F}_{q^{2}}^{*} \oplus G L_{2}\left(\mathbb{F}_{q}\right)^{8} \oplus G L_{3}\left(\mathbb{F}_{q}\right)^{4} \oplus G L_{3}\left(\mathbb{F}_{q^{2}}\right)^{2}
$$

4. for $p^{k} \equiv 11 \bmod 12$ with $k$ odd, we have

$$
U\left(\mathbb{F}_{q} G_{1}\right) \cong\left(\mathbb{F}_{q}^{*}\right)^{2} \oplus \mathbb{F}_{q^{2}}^{*} \oplus G L_{2}\left(\mathbb{F}_{q}\right)^{8} \oplus G L_{3}\left(\mathbb{F}_{q^{2}}\right)^{4}
$$

Proof. Since $\mathbb{F}_{q} G_{1}$ is semisimple, using Lemma 2.3 we get

$$
\mathbb{F}_{q} G_{1} \cong \mathbb{F}_{q} \oplus_{r=1}^{t-1} M_{n_{r}}\left(\mathbb{F}_{r}\right), \text { for some } t \in \mathbb{Z} \text {. }
$$

First assume that $k$ is even which means for any prime $p>3$, we have

$$
p^{k} \equiv 1 \bmod 3 \text { and } p^{k} \equiv 1 \bmod 4 .
$$

Using Chinese remainder theorem, we get $p^{k} \equiv 1 \bmod 12$. This means $\left|S\left(\gamma_{g}\right)\right|=1$ for each $g \in G_{1}$ as $I_{\mathbb{F}}=\{1\}$. Hence, (1), Theorems 2.1 and 2.2 imply that

$$
\mathbb{F}_{q} G_{1} \cong \mathbb{F}_{q} \oplus_{r=1}^{19} M_{n_{r}}\left(\mathbb{F}_{q}\right) \text {. }
$$

Incorporating Theorem 2.5 with $G_{1}^{\prime} \cong\left(C_{3} \times C_{3}\right) \rtimes C_{3}$ in (2) to obtain

$$
\mathbb{F}_{q} G_{1} \cong \mathbb{F}_{q}^{4} \oplus_{r=1}^{16} M_{n_{r}}\left(\mathbb{F}_{q}\right), \text { where } n_{r} \geq 2 \text { with } 104=\sum_{r=1}^{16} n_{r}^{2} .
$$

Above equation gives the only possibility $\left(2^{8}, 3^{8}\right)$ for the values of $n_{r}^{\prime}$ s where $a^{b}$ means $(a, a, \cdots, b$ times $)$ and therefore, (3) implies that

$$
\mathbb{F}_{q} G_{1} \cong \mathbb{F}_{q}^{4} \oplus M_{2}\left(\mathbb{F}_{q}\right)^{8} \oplus M_{3}\left(\mathbb{F}_{q}\right)^{8}
$$

Now we consider that $k$ is odd. We shall discuss this possibility in the following four cases: Case 1: $p^{k} \equiv 1 \bmod 3$ and $p^{k} \equiv 1 \bmod 4$. In this case, Wedderburn decomposition is given by (4). Case 2. $p^{k} \equiv 5 \bmod 12$. In this case, we have

$$
S\left(\gamma_{t}\right)=\left\{\gamma_{t}, \gamma_{t^{2}}\right\}, S\left(\gamma_{x t}\right)=\left\{\gamma_{x t}, \gamma_{x t^{2}}\right\}, S\left(\gamma_{y t}\right)=\left\{\gamma_{y t}, \gamma_{y t^{2}}\right\}, S\left(\gamma_{x y t}\right)=\left\{\gamma_{x y t}, \gamma_{x y t^{2}}\right\},
$$


and $S\left(\gamma_{g}\right)=\left\{\gamma_{g}\right\}$ for the remaining representatives $g$ of conjugacy classes. Therefore, (1) and Theorems $2.1,2.2$ imply that

$$
\mathbb{F}_{q} G_{1} \cong \mathbb{F}_{q} \oplus_{r=1}^{11} M_{n_{r}}\left(\mathbb{F}_{q}\right) \oplus_{r=12}^{15} M_{n_{r}}\left(\mathbb{F}_{q^{2}}\right) \text {. }
$$

Since $G_{1} / G_{1}^{\prime} \cong C_{4}$, we have $\mathbb{F}_{q} C_{4} \cong \mathbb{F}_{q}^{4}$. This with above and Theorem 2.5 yields

$$
\mathbb{F}_{q} G_{1} \cong \mathbb{F}_{q}^{4} \oplus_{r=1}^{8} M_{n_{r}}\left(\mathbb{F}_{q}\right) \oplus_{r=9}^{12} M_{n_{r}}\left(\mathbb{F}_{q^{2}}\right), n_{r} \geq 2 \text { with } 104=\sum_{r=1}^{8} n_{r}^{2}+2 \sum_{r=9}^{12} n_{r}^{2}
$$

Further, consider the normal subgroup $H_{1}=\langle t\rangle$ of $G_{1}$ having order 3 with $K_{1}=G_{1} / H_{1} \cong\left(C_{3} \times\right.$ $\left.C_{3}\right) \rtimes C_{4}$. The quotient group $K_{1}$ has 12 conjugacy classes as shown in the table below. Here elements of $K_{1}$ are cosets, for instance, $x \in K_{1}$ is $x H_{1}$ but we keep the same notation.

\begin{tabular}{|c|c|c|c|c|c|c|c|c|c|c|c|c|}
\hline $\mathrm{r}$ & $e$ & $x$ & $y$ & $z$ & $w$ & $x y$ & $y z$ & $y w$ & $z w$ & $y z w$ & $z^{2} w$ & $y z^{2} w$ \\
\hline $\mathrm{s}$ & 1 & 9 & 1 & 2 & 2 & 9 & 2 & 2 & 2 & 2 & 2 & 2 \\
\hline $\mathrm{o}$ & 1 & 4 & 2 & 3 & 3 & 4 & 6 & 6 & 3 & 6 & 3 & 6 \\
\hline
\end{tabular}

It can be verified that for all the representatives $g$ of $K_{1},\left|S\left(\gamma_{g}\right)\right|=1$. Therefore, from Theorems 2.1 and 2.2 , we have

$$
\mathbb{F}_{q} K_{1} \cong \mathbb{F}_{q} \oplus_{r=1}^{11} M_{t_{r}}\left(\mathbb{F}_{q}\right), t_{r} \in \mathbb{Z}
$$

Observe that $K_{1} / K_{1}^{\prime} \cong C_{4}$. So, Theorem 2.5 implies that

$$
\mathbb{F}_{q} K_{1} \cong \mathbb{F}_{q}^{4} \oplus_{r=1}^{8} M_{t_{r}}\left(\mathbb{F}_{q}\right), \text { with } 32=\sum_{r=1}^{8} t_{r}^{2}, t_{r} \geq 2 .
$$

This gives us the only choice $\left(2^{8}\right)$ for values of $t_{r}^{\prime} \mathrm{s}$ and therefore, Theorem 2.5 and (5) yields

$$
\mathbb{F}_{q} G_{1} \cong \mathbb{F}_{q}^{4} \oplus M_{2}\left(\mathbb{F}_{q}\right)^{8} \oplus_{r=1}^{4} M_{n_{r}}\left(\mathbb{F}_{q^{2}}\right), n_{r} \geq 2 \text { with } 36=\sum_{r=1}^{4} n_{r}^{2}
$$

Above leaves us with the only choice $\left(3^{4}\right)$ for values of $n_{r}^{\prime}$ s which means the required Wedderburn decomposition is

$$
\mathbb{F}_{q} G_{1} \cong \mathbb{F}_{q}^{4} \oplus M_{2}\left(\mathbb{F}_{q}\right)^{8} \oplus M_{3}\left(\mathbb{F}_{q^{2}}\right)^{4}
$$

Case 3. $p^{k} \equiv 7 \bmod 12$. In this case, we have

$$
S\left(\gamma_{x}\right)=\left\{\gamma_{x}, \gamma_{x y}\right\}, S\left(\gamma_{x t}\right)=\left\{\gamma_{x t}, \gamma_{x y t}\right\}, S\left(\gamma_{x t^{2}}\right)=\left\{\gamma_{x t^{2}}, \gamma_{x y t^{2}}\right\}, \text { and } S\left(\gamma_{g}\right)=\left\{\gamma_{g}\right\}
$$

for the remaining representatives $g$ of conjugacy classes. Therefore (1) and Theorems 2.1, 2.2 imply that

$$
\mathbb{F}_{q} G_{1} \cong \mathbb{F}_{q} \oplus_{r=1}^{13} M_{n_{r}}\left(\mathbb{F}_{q}\right) \oplus_{r=14}^{16} M_{n_{r}}\left(\mathbb{F}_{q^{2}}\right) \text {. }
$$

Since $G_{1} / G_{1}^{\prime} \cong C_{4}$, we have $\mathbb{F}_{q} C_{4} \cong \mathbb{F}_{q}^{2} \oplus \mathbb{F}_{q^{2}}$. This with above and Theorem 2.5 yields

$$
\mathbb{F}_{q} G_{1} \cong \mathbb{F}_{q}^{2} \oplus \mathbb{F}_{q^{2}} \oplus_{r=1}^{12} M_{n_{r}}\left(\mathbb{F}_{q}\right) \oplus_{r=13}^{14} M_{n_{r}}\left(\mathbb{F}_{q^{2}}\right), n_{r} \geq 2 \text { with } 104=\sum_{r=1}^{12} n_{r}^{2}+2 \sum_{r=13}^{14} n_{r}^{2}
$$


Again consider the normal subgroup $H_{1}$ of $G_{1}$. In this case, it can be verified that $\left|S\left(\gamma_{g}\right)\right|=1$ for all the representatives $g$ of $K_{1}$ except $x$ and $x y$ for which $S\left(\gamma_{x}\right)=\left\{\gamma_{x}, \gamma_{x y}\right\}$. Therefore, employing Theorems 2.1 and 2.2 to obtain

$$
\mathbb{F}_{q} K_{1} \cong \mathbb{F}_{q} \oplus_{r=1}^{9} M_{t_{r}}\left(\mathbb{F}_{q}\right) \oplus M_{t_{10}}\left(\mathbb{F}_{q^{2}}\right), t_{r} \in \mathbb{Z}
$$

Since $K_{1} / K_{1}^{\prime} \cong C_{4}$, above and Theorem 2.5 imply that

$$
\mathbb{F}_{q} K_{1} \cong \mathbb{F}_{q}^{2} \oplus \mathbb{F}_{q^{2}} \oplus_{r=1}^{8} M_{t_{r}}\left(\mathbb{F}_{q}\right), \text { with } 32=\sum_{r=1}^{8} t_{r}^{2}, t_{r} \geq 2 .
$$

This gives us the only choice $\left(2^{8}\right)$ for values of $t_{r}^{\prime}$ s. Hence, Theorem 2.6 and (6) imply that

$$
\mathbb{F}_{q} G_{1} \cong \mathbb{F}_{q}^{2} \oplus \mathbb{F}_{q^{2}} \oplus M_{2}\left(\mathbb{F}_{q}\right)^{8} \oplus_{r=1}^{4} M_{n_{r}}\left(\mathbb{F}_{q}\right) \oplus_{r=5}^{6} M_{n_{r}}\left(\mathbb{F}_{q^{2}}\right), \text { with } 72=\sum_{r=1}^{4} n_{r}^{2}+2 \sum_{r=5}^{6} n_{r}^{2}
$$

Above leaves us with the only choice $\left(3^{6}\right)$ for values of $n_{r}^{\prime}$ s which means the required Wedderburn decomposition is

$$
\mathbb{F}_{q} G_{1} \cong \mathbb{F}_{q}^{2} \oplus \mathbb{F}_{q^{2}} \oplus M_{2}\left(\mathbb{F}_{q}\right)^{8} \oplus M_{3}\left(\mathbb{F}_{q}\right)^{4} \oplus M_{3}\left(\mathbb{F}_{q^{2}}\right)^{2} .
$$

Case 4. $p^{k} \equiv 11 \bmod 12$. In this case, we have

$$
\begin{gathered}
S\left(\gamma_{t}\right)=\left\{\gamma_{t}, \gamma_{t^{2}}\right\}, S\left(\gamma_{x t}\right)=\left\{\gamma_{x t}, \gamma_{x y t^{2}}\right\}, S\left(\gamma_{y t}\right)=\left\{\gamma_{y t}, \gamma_{y t^{2}}\right\}, S\left(\gamma_{x y t}\right)=\left\{\gamma_{x y t}, \gamma_{x t^{2}}\right\}, \\
S\left(\gamma_{x}\right)=\left\{\gamma_{x}, \gamma_{x y}\right\}, \text { and } S\left(\gamma_{g}\right)=\left\{\gamma_{g}\right\}
\end{gathered}
$$

for the remaining representatives $g$ of conjugacy classes. Therefore, (1) and Theorems 2.1, 2.2 imply that

$$
\mathbb{F}_{q} G_{1} \cong \mathbb{F}_{q} \oplus_{r=1}^{9} M_{n_{r}}\left(\mathbb{F}_{q}\right) \oplus_{r=10}^{14} M_{n_{r}}\left(\mathbb{F}_{q^{2}}\right) .
$$

Since $G_{1} / G_{1}^{\prime} \cong C_{4}$, we have $\mathbb{F}_{q} C_{4} \cong \mathbb{F}_{q}^{2} \oplus \mathbb{F}_{q^{2}}$. This with above and Theorem 2.5 yields

$$
\mathbb{F}_{q} G_{1} \cong \mathbb{F}_{q}^{2} \oplus \mathbb{F}_{q^{2}} \oplus_{r=1}^{8} M_{n_{r}}\left(\mathbb{F}_{q}\right) \oplus_{r=9}^{12} M_{n_{r}}\left(\mathbb{F}_{q^{2}}\right), n_{r} \geq 2 \text { with } 104=\sum_{r=1}^{8} n_{r}^{2}+2 \sum_{r=9}^{12} n_{r}^{2}
$$

In this case, again we have $\left|S\left(\gamma_{g}\right)\right|=1$ for all representatives $g$ of $K_{1}$ except $x$ and $x y$ which means the Wedderburn decomposition of $\mathbb{F}_{q} K_{1}$ is same as obtained in case 3, i.e.

$$
\mathbb{F}_{q} K_{1} \cong \mathbb{F}_{q}^{2} \oplus \mathbb{F}_{q^{2}} \oplus M_{2}\left(\mathbb{F}_{q}\right)^{8} .
$$

Now employ Theorem 2.6 and (7) to obtain

$$
\mathbb{F}_{q} G_{1} \cong \mathbb{F}_{q}^{2} \oplus \mathbb{F}_{q^{2}} \oplus M_{2}\left(\mathbb{F}_{q}\right)^{8} \oplus_{r=1}^{4} M_{n_{r}}\left(\mathbb{F}_{q^{2}}\right), \text { with } 36=\sum_{r=1}^{4} n_{r}^{2} .
$$

This leaves us with the only choice $\left(3^{4}\right)$ for values of $n_{r}^{\prime}$ s which means the required Wedderburn decomposition is

$$
\mathbb{F}_{q} G_{1} \cong \mathbb{F}_{q}^{2} \oplus \mathbb{F}_{q^{2}} \oplus M_{2}\left(\mathbb{F}_{q}\right)^{8} \oplus M_{3}\left(\mathbb{F}_{q^{2}}\right)^{4}
$$




\subsection{The group $G_{2}=\left(\left(C_{3} \times C_{3}\right) \rtimes C_{3}\right) \rtimes C_{4}$}

Group $G_{2}$ has the following presentation:

$$
\begin{gathered}
G_{2}=\langle x, y, z, w, t| x^{2} y^{-1},[y, x],[z, x] w^{-2},[w, x] t^{-1} w^{-1} z^{-2},[t, x], y^{2},[z, y] z^{-1}, \\
\left.[w, y] t^{-1} w^{-1},[t, y], z^{3},[w, z] t^{-1},[t, z], w^{3},[t, w], t^{3}\right\rangle .
\end{gathered}
$$

\begin{tabular}{|c|c|c|c|c|c|c|c|c|c|c|c|c|}
\hline$e$ & $\begin{array}{lll}x & y\end{array}$ & $z$ & $w$ & & $x y$ & $x z$ & $x t$ & $y w$ & & & $x y z$ & xyz \\
\hline \begin{tabular}{c|c}
$\mathrm{s}$ & 1 \\
\end{tabular} & \begin{tabular}{l|l|l}
99 \\
\end{tabular} & 12 & 12 & & 9 & 9 & 0 & 9 & 9 & & & \\
\hline \begin{tabular}{l|l} 
o & 1
\end{tabular} & & & 3 & & 4 & 12 & 12 & 6 & & & 12 & 12 \\
\hline
\end{tabular}

Further, $G_{2}$ has 14 conjugacy classes as shown in the table below.

From above discussion, clearly the exponent of $G_{2}$ is 12 . Also $G_{2}^{\prime} \cong\left(C_{3} \times C_{3}\right) \rtimes C_{3}$ and $G_{2} / G_{2}^{\prime} \cong C_{4}$. Next, we discuss the unit group of $\mathbb{F}_{q} G_{2}$ when $p>3$.

Theorem 3.2. The unit group of $\mathbb{F}_{q} G_{2}$, for $q=p^{k}, p>3$ where $\mathbb{F}_{q}$ is a finite field having $q=p^{k}$ elements is as follows:

1. for any $p$ and $k$ even or $p^{k} \equiv 1 \bmod 12$ with $k$ odd, we have

$$
U\left(\mathbb{F}_{q} G_{2}\right) \cong\left(\mathbb{F}_{q}^{*}\right)^{4} \oplus G L_{3}\left(\mathbb{F}_{q}\right)^{8} \oplus G L_{4}\left(\mathbb{F}_{q}\right)^{2} .
$$

2. for $p^{k} \equiv 5 \bmod 12$ with $k$ odd, we have

$$
U\left(\mathbb{F}_{q} G_{2}\right) \cong\left(\mathbb{F}_{q}^{*}\right)^{4} \oplus G L_{4}\left(\mathbb{F}_{q}\right)^{2} \oplus G L_{3}\left(\mathbb{F}_{q^{2}}\right)^{4} .
$$

3. for $p^{k} \equiv 7 \bmod 12$ with $k$ odd, we have

$$
U\left(\mathbb{F}_{q} G_{2}\right) \cong\left(\mathbb{F}_{q}^{*}\right)^{2} \oplus \mathbb{F}_{q^{2}}^{*} \oplus G L_{3}\left(\mathbb{F}_{q}\right)^{4} \oplus G L_{4}\left(\mathbb{F}_{q}\right)^{2} \oplus G L_{3}\left(\mathbb{F}_{q^{2}}\right)^{2} .
$$

4. for $p^{k} \equiv 11$ mod 12 with $k$ odd, we have

$$
U\left(\mathbb{F}_{q} G_{2}\right) \cong\left(\mathbb{F}_{q}^{*}\right)^{2} \oplus \mathbb{F}_{q^{2}}^{*} \oplus G L_{4}\left(\mathbb{F}_{q}\right)^{2} \oplus G L_{3}\left(\mathbb{F}_{q^{2}}\right)^{4} .
$$

Proof. Since $\mathbb{F}_{q} G_{2}$ is semisimple, we have

$$
\mathbb{F}_{q} G_{2} \cong \mathbb{F}_{q} \oplus_{r=1}^{t-1} M_{n_{r}}\left(\mathbb{F}_{r}\right) \text {, for some } t \in \mathbb{Z} .
$$

First assume that $k$ is even which means for any prime $p>3, p^{k} \equiv 1 \bmod 12$. This means $\left|S\left(\gamma_{g}\right)\right|=1$ for each $g \in G_{2}$. Hence, (8), Theorems 2.1 and 2.2 imply that

$$
\mathbb{F}_{q} G_{2} \cong \mathbb{F}_{q} \oplus_{r=1}^{13} M_{n_{r}}\left(\mathbb{F}_{q}\right) .
$$

Using Theorem 2.5 with $G_{2}^{\prime} \cong\left(C_{3} \times C_{3}\right) \rtimes C_{3}$ to obtain

$$
\mathbb{F}_{q} G_{2} \cong \mathbb{F}_{q}^{4} \oplus_{r=1}^{10} M_{n_{r}}\left(\mathbb{F}_{q}\right), \text { where } n_{r} \geq 2 \text { with } 104=\sum_{r=1}^{10} n_{r}^{2} .
$$

Above equation gives us four possibilities $\left(2^{8}, 6^{2}\right),\left(2^{5}, 3^{2}, 4,5^{2}\right),\left(2^{4}, 3^{4}, 4,6\right)$ and $\left(3^{8}, 4^{2}\right)$ for the values of $n_{r}^{\prime}$ s. Further, consider the normal subgroup $H_{2}=\langle t\rangle$ of $G_{2}$ having order 3 with $K_{2}=G_{2} / H_{2} \cong$ $\left(C_{3} \times C_{3}\right) \rtimes C_{4}$. It can be verified that $K_{2}$ has 6 conjugacy classes as shown in the table below.

$$
\begin{array}{|c|c|c|c|c|c|c|}
\hline \mathrm{r} & e & x & y & z & w & x y \\
\hline \mathrm{s} & 1 & 9 & 9 & 4 & 4 & 9 \\
\hline \mathrm{o} & 1 & 4 & 2 & 3 & 3 & 4 \\
\hline
\end{array}
$$


Also, for all the representatives $g$ of $K_{2},\left|S\left(\gamma_{g}\right)\right|=1$ which means by Theorems 2.1 and 2.2, we have

$$
\mathbb{F}_{q} K_{2} \cong \mathbb{F}_{q} \oplus_{r=1}^{5} M_{t_{r}}\left(\mathbb{F}_{q}\right), t_{r} \in \mathbb{Z}
$$

Observe that $K_{2} / K_{2}^{\prime} \cong C_{4}$. This with above and Theorem 2.4 imply that

$$
\mathbb{F}_{q} K_{2} \cong \mathbb{F}_{q}^{4} \oplus_{r=1}^{2} M_{t_{r}}\left(\mathbb{F}_{q}\right), \text { with } 32=\sum_{r=1}^{2} t_{r}^{2}, t_{r} \geq 2
$$

This gives us the only choice $\left(4^{2}\right)$ for values of $t_{r}^{\prime}$ s. Therefore, Theorem 2.6 and (9) imply that $\left(3^{8}, 4^{2}\right)$ is the correct choice for values of $n_{r}^{\prime} \mathrm{s}$ and therefore, we have

$$
\mathbb{F}_{q} G_{2} \cong \mathbb{F}_{q}^{4} \oplus M_{3}\left(\mathbb{F}_{q}\right)^{8} \oplus M_{4}\left(\mathbb{F}_{q}\right)^{2}
$$

Now we consider that $k$ is odd. We shall discuss this possibility in the following four cases:

Case 1: $p^{k} \equiv 1 \bmod 12$. In this case, Wedderburn decomposition is given by (10).

Case 2. $p^{k} \equiv 5 \bmod 12$. In this case, we have

$$
S\left(\gamma_{t}\right)=\left\{\gamma_{t}, \gamma_{t^{2}}\right\}, S\left(\gamma_{x z}\right)=\left\{\gamma_{x z}, \gamma_{x t}\right\}, S\left(\gamma_{y w}\right)=\left\{\gamma_{y w}, \gamma_{y t}\right\}, S\left(\gamma_{x y z}\right)=\left\{\gamma_{x y z}, \gamma_{x y z w}\right\},
$$

and $S\left(\gamma_{g}\right)=\left\{\gamma_{g}\right\}$ for the remaining representatives $g$ of conjugacy classes. Therefore, (8) and Theorems 2.1, 2.2 imply that

$$
\mathbb{F}_{q} G_{2} \cong \mathbb{F}_{q} \oplus_{r=1}^{5} M_{n_{r}}\left(\mathbb{F}_{q}\right) \oplus_{r=6}^{9} M_{n_{r}}\left(\mathbb{F}_{q^{2}}\right) .
$$

Since $G_{2} / G_{2}^{\prime} \cong C_{4}$, we have $\mathbb{F}_{q} C_{4} \cong \mathbb{F}_{q}^{4}$. This with above and Theorem 2.5 yields

$$
\mathbb{F}_{q} G_{2} \cong \mathbb{F}_{q}^{4} \oplus_{r=1}^{2} M_{n_{r}}\left(\mathbb{F}_{q}\right) \oplus_{r=3}^{6} M_{n_{r}}\left(\mathbb{F}_{q^{2}}\right), n_{r} \geq 2 \text { with } 104=\sum_{r=1}^{2} n_{r}^{2}+2 \sum_{r=3}^{6} n_{r}^{2}
$$

Further, again consider the normal subgroup $H_{2}=\langle t\rangle$ of $G_{2}$. It can be verified that for all the representatives $g$ of $K_{2},\left|S\left(\gamma_{g}\right)\right|=1$ which means (as earlier)

$$
\mathbb{F}_{q} K_{2} \cong \mathbb{F}_{q}^{4} \oplus M_{4}\left(\mathbb{F}_{q}\right)^{2}
$$

This with Theorem 2.6 and (11) imply that

$$
\mathbb{F}_{q} G_{2} \cong \mathbb{F}_{q}^{4} \oplus M_{4}\left(\mathbb{F}_{q}\right)^{2} \oplus_{r=1}^{4} M_{n_{r}}\left(\mathbb{F}_{q^{2}}\right), n_{r} \geq 2 \text { with } 36=\sum_{r=1}^{4} n_{r}^{2}
$$

Above leaves us with the only choice $\left(3^{4}\right)$ for values of $n_{r}^{\prime}$ s which means the required Wedderburn decomposition is

$$
\mathbb{F}_{q} G_{2} \cong \mathbb{F}_{q}^{4} \oplus M_{4}\left(\mathbb{F}_{q}\right)^{2} \oplus M_{3}\left(\mathbb{F}_{q^{2}}\right)^{4} .
$$

Case 3. $p^{k} \equiv 7 \bmod 12$. In this case, we have

$$
S\left(\gamma_{x}\right)=\left\{\gamma_{x}, \gamma_{x y}\right\}, S\left(\gamma_{x z}\right)=\left\{\gamma_{x z}, \gamma_{x y z w}\right\}, S\left(\gamma_{x t}\right)=\left\{\gamma_{x t}, \gamma_{x y z}\right\}, \text { and } S\left(\gamma_{g}\right)=\left\{\gamma_{g}\right\}
$$

for the remaining representatives $g$ of conjugacy classes. Therefore, (8) and Theorems 2.1, 2.2 imply that

$$
\mathbb{F}_{q} G_{2} \cong \mathbb{F}_{q} \oplus_{r=1}^{7} M_{n_{r}}\left(\mathbb{F}_{q}\right) \oplus_{r=8}^{10} M_{n_{r}}\left(\mathbb{F}_{q^{2}}\right) .
$$

Since $G_{2} / G_{2}^{\prime} \cong C_{4}$, we have $\mathbb{F}_{q} C_{4} \cong \mathbb{F}_{q}^{2} \oplus \mathbb{F}_{q^{2}}$. This with Theorem 2.5 yields

$$
\mathbb{F}_{q} G_{2} \cong \mathbb{F}_{q}^{2} \oplus \mathbb{F}_{q^{2}} \oplus_{r=1}^{6} M_{n_{r}}\left(\mathbb{F}_{q}\right) \oplus_{r=7}^{8} M_{n_{r}}\left(\mathbb{F}_{q^{2}}\right), n_{r} \geq 2 \text { with } 104=\sum_{r=1}^{6} n_{r}^{2}+2 \sum_{r=7}^{8} n_{r}^{2}
$$


Further, it can be verified that $\left|S\left(\gamma_{g}\right)\right|=1$ for all the representatives $g$ of $K_{2}$ except $x$ and $x y$. For these, we have $S\left(\gamma_{x}\right)=\left\{\gamma_{x}, \gamma_{x y}\right\}$ which means by Theorems 2.1 and 2.2 , we have

$$
\mathbb{F}_{q} K_{2} \cong \mathbb{F}_{q} \oplus_{r=1}^{3} M_{t_{r}}\left(\mathbb{F}_{q}\right) \oplus M_{t_{4}}\left(\mathbb{F}_{q^{2}}\right), t_{r} \in \mathbb{Z}
$$

Incorporating $K_{2} / K_{2}^{\prime} \cong C_{4}$ with Theorem 2.5 to obtain

$$
\mathbb{F}_{q} K_{2} \cong \mathbb{F}_{q}^{2} \oplus \mathbb{F}_{q^{2}} \oplus_{r=1}^{2} M_{t_{r}}\left(\mathbb{F}_{q}\right), \text { with } 32=\sum_{r=1}^{2} t_{r}^{2}, t_{r} \geq 2
$$

This gives us only choice $\left(4^{2}\right)$ for values of $t_{r}^{\prime}$ s. Therefore, Theorem 2.6 and (12) yields

$$
\mathbb{F}_{q} G_{2} \cong \mathbb{F}_{q}^{2} \oplus \mathbb{F}_{q^{2}} \oplus M_{4}\left(\mathbb{F}_{q}\right)^{2} \oplus_{r=1}^{4} M_{n_{r}}\left(\mathbb{F}_{q}\right) \oplus_{r=5}^{6} M_{n_{r}}\left(\mathbb{F}_{q^{2}}\right), \text { with } 72=\sum_{r=1}^{4} n_{r}^{2}+2 \sum_{r=5}^{6} n_{r}^{2}
$$

Above leaves us with the only choice $\left(3^{6}\right)$ for values of $n_{r}^{\prime}$ which means the required Wedderburn decomposition is

$$
\mathbb{F}_{q} G_{2} \cong \mathbb{F}_{q}^{2} \oplus \mathbb{F}_{q^{2}} \oplus M_{4}\left(\mathbb{F}_{q}\right)^{2} \oplus M_{3}\left(\mathbb{F}_{q}\right)^{4} \oplus M_{3}\left(\mathbb{F}_{q^{2}}\right)^{2}
$$

Case 4. $p^{k} \equiv 11 \bmod 12$. In this case, we have

$$
\begin{gathered}
S\left(\gamma_{t}\right)=\left\{\gamma_{t}, \gamma_{t^{2}}\right\}, S\left(\gamma_{x}\right)=\left\{\gamma_{x}, \gamma_{x y}\right\}, S\left(\gamma_{x z}\right)=\left\{\gamma_{x z}, \gamma_{x y z}\right\}, S\left(\gamma_{x t}\right)=\left\{\gamma_{x t}, \gamma_{x y z w}\right\} \\
S\left(\gamma_{y w}\right)=\left\{\gamma_{y w}, \gamma_{y t}\right\}, \text { and } S\left(\gamma_{g}\right)=\left\{\gamma_{g}\right\}
\end{gathered}
$$

for the remaining representatives $g$ of conjugacy classes. Therefore, (8) and Theorems 2.1, 2.2 imply that

$$
\mathbb{F}_{q} G_{2} \cong \mathbb{F}_{q} \oplus_{r=1}^{3} M_{n_{r}}\left(\mathbb{F}_{q}\right) \oplus_{r=4}^{8} M_{n_{r}}\left(\mathbb{F}_{q^{2}}\right) .
$$

Since $G_{2} / G_{2}^{\prime} \cong C_{4}$, we have $\mathbb{F}_{q} C_{4} \cong \mathbb{F}_{q}^{2} \oplus \mathbb{F}_{q^{2}}$. This with above and Theorem 2.5 yields

$$
\mathbb{F}_{q} G_{2} \cong \mathbb{F}_{q}^{2} \oplus \mathbb{F}_{q^{2}} \oplus_{r=1}^{2} M_{n_{r}}\left(\mathbb{F}_{q}\right) \oplus_{r=3}^{6} M_{n_{r}}\left(\mathbb{F}_{q^{2}}\right), n_{r} \geq 2 \text { with } 104=\sum_{r=1}^{2} n_{r}^{2}+2 \sum_{r=3}^{6} n_{r}^{2}
$$

Further, it can be verified that $\left|S\left(\gamma_{g}\right)\right|=1$ for all the representatives $g$ of $K_{2}$ except $x$ and $x y$ which means (as in case 3),

$$
\mathbb{F}_{q} K_{2} \cong \mathbb{F}_{q}^{2} \oplus \mathbb{F}_{q^{2}} \oplus M_{4}\left(\mathbb{F}_{q}\right)^{2}
$$

Now employ Theorem 2.6 and (13) to obtain

$$
\mathbb{F}_{q} G_{2} \cong \mathbb{F}_{q}^{2} \oplus \mathbb{F}_{q^{2}} \oplus M_{4}\left(\mathbb{F}_{q}\right)^{2} \oplus_{r=1}^{4} M_{n_{r}}\left(\mathbb{F}_{q^{2}}\right), \text { with } 36=\sum_{r=1}^{4} n_{r}^{2}
$$

Above leaves us with the only choice $\left(3^{4}\right)$ for values of $n_{r}^{\prime}$ which means the required Wedderburn decomposition is

$$
\mathbb{F}_{q} G_{2} \cong \mathbb{F}_{q}^{2} \oplus \mathbb{F}_{q^{2}} \oplus M_{4}\left(\mathbb{F}_{q}\right)^{2} \oplus M_{3}\left(\mathbb{F}_{q^{2}}\right)^{4}
$$




\subsection{The group $G_{3}=\left(\left(C_{3} \times C_{3}\right) \rtimes C_{3}\right) \rtimes\left(C_{2} \times C_{2}\right)$}

Group $G_{3}$ has the following presentation:

$$
\begin{gathered}
G_{3}=\langle x, y, z, w, t| x^{2},[y, x],[z, x],[w, x] w^{-1},[t, x] t^{-1}, y^{2},[z, y] z^{-1},[w, y],[t, y] t^{-1}, \\
\left.z^{3},[w, z] t^{-1},[t, z], w^{3},[t, w], t^{3}\right\rangle .
\end{gathered}
$$

\begin{tabular}{|c|c|c|c|c|c|c|c|c|c|}
\hline & \begin{tabular}{l|l}
1 & $x$ \\
\end{tabular} & $u z$ & 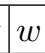 & & & & $y w$ & $z u$ & \\
\hline & \begin{tabular}{|l|l|l}
1 &
\end{tabular} & 96 & 6 & & 9 & 18 & 18 & 12 & 18 \\
\hline & \begin{tabular}{|l|l|}
1 &
\end{tabular} & \begin{tabular}{l|l|l}
2 & 3
\end{tabular} & 3 & & 2 & & 6 & & \\
\hline
\end{tabular}

Further, $G_{3}$ has 11 conjugacy classes as shown in the table below.

From above discussion, clearly the exponent of $G_{3}$ is 6 . Also $G_{3}^{\prime} \cong\left(C_{3} \times C_{3}\right) \rtimes C_{3}$ with $G_{3} / G_{3}^{\prime} \cong C_{2} \times C_{2}$. Next, we discuss the unit group of $\mathbb{F}_{q} G_{3}$ when $p>3$.

Theorem 3.3. The unit group of $\mathbb{F}_{q} G_{3}$, for $q=p^{k}, p>3$ where $\mathbb{F}_{q}$ is a finite field having $q=p^{k}$ elements is as follows:

$$
U\left(\mathbb{F}_{q} G_{3}\right) \cong\left(\mathbb{F}_{q}^{*}\right)^{4} \oplus G L_{2}\left(\mathbb{F}_{q}\right)^{4} \oplus G L_{4}\left(\mathbb{F}_{q}\right) \oplus G L_{6}\left(\mathbb{F}_{q}\right)^{2} .
$$

Proof. Since $\mathbb{F}_{q} G_{3}$ is semisimple, we have

$$
\mathbb{F}_{q} G_{3} \cong \mathbb{F}_{q} \oplus_{r=1}^{t-1} M_{n_{r}}\left(\mathbb{F}_{r}\right) \text {, for some } t \in \mathbb{Z} .
$$

First assume that $k$ is even which means for any prime $p>3, p^{k} \equiv 1 \bmod 6$. This means $\left|S\left(\gamma_{g}\right)\right|=1$ for each $g \in G_{3}$. Hence, (14), Theorems 2.1 and 2.2 imply that

$$
\mathbb{F}_{q} G_{3} \cong \mathbb{F}_{q} \oplus_{r=1}^{10} M_{n_{r}}\left(\mathbb{F}_{q}\right) .
$$

Incorporating Theorem 2.5 with $G_{3}^{\prime} \cong\left(C_{3} \times C_{3}\right) \rtimes C_{3}$ in above to obtain

$$
\mathbb{F}_{q} G_{3} \cong \mathbb{F}_{q}^{4} \oplus_{r=1}^{7} M_{n_{r}}\left(\mathbb{F}_{q}\right), \text { where } n_{r} \geq 2 \text { with } 104=\sum_{r=1}^{7} n_{r}^{2} .
$$

\begin{tabular}{|c|c|c|c|c|c|c|c|}
\hline & $x \mid y$ & & & & & $y w$ & \\
\hline & & 2 & 2 & 9 & 6 & 6 & \\
\hline & & & & 2 & 6 & 6 & \\
\hline
\end{tabular}

Above equation gives us four possibilities $\left(2^{4}, 4,6^{2}\right),\left(2^{3}, 3^{2}, 5,7\right),\left(2,3^{2}, 4^{2}, 5^{2}\right)$ and $\left(3^{4}, 4^{2}, 6\right)$ for the values of $n_{r}^{\prime}$ s. Further, consider the normal subgroup $H_{3}=\langle t\rangle$ of $G_{3}$ having order 3 with $K_{3}=G_{3} / H_{3} \cong$ $S_{3} \times S_{3}$. It can be verified that $K_{3}$ has 9 conjugacy classes as shown in the table below.

Further, for all the representatives $g$ of $K_{3},\left|S\left(\gamma_{g}\right)\right|=1$ which means by Theorems 2.1 and 2.2, we have

$$
\mathbb{F}_{q} K_{3} \cong \mathbb{F}_{q} \oplus_{r=1}^{8} M_{t_{r}}\left(\mathbb{F}_{q}\right), t_{r} \in \mathbb{Z} .
$$

Observe that $K_{3} / K_{3}^{\prime} \cong C_{2} \times C_{2}$. So, above and Theorem 2.5 imply that

$$
\mathbb{F}_{q} K_{3} \cong \mathbb{F}_{q}^{4} \oplus_{r=1}^{5} M_{t_{r}}\left(\mathbb{F}_{q}\right), \text { with } 32=\sum_{r=1}^{5} t_{r}^{2}, t_{r} \geq 2
$$


This gives us the only choice $\left(2^{4}, 4\right)$ for values of $t_{r}^{\prime}$ s. Therefore, from Theorem 2.6 and $(15)$, we conclude that $\left(2^{4}, 4,6^{2}\right)$ is the correct choice for $n_{r}^{\prime}$ s which means

$$
\mathbb{F}_{q} G_{3} \cong \mathbb{F}_{q}^{4} \oplus M_{2}\left(\mathbb{F}_{q}\right)^{4} \oplus M_{4}\left(\mathbb{F}_{q}\right) \oplus M_{6}\left(\mathbb{F}_{q}\right)^{2}
$$

Now we consider that $k$ is odd. We shall discuss this possibility in the following two cases: Case 1: $p^{k} \equiv 1 \bmod 6$. In this case, Wedderburn decomposition is given by (16).

Case 2. $p^{k} \equiv 5 \bmod 6$. In this case, we have $S\left(\gamma_{g}\right)=\left\{\gamma_{g}\right\}$ for all the representatives $g$ of conjugacy classes. Therefore, Wedderburn decomposition is again given by (16).

\subsection{The group $G_{4}=C_{2} \times\left(\left(\left(C_{3} \times C_{3}\right) \rtimes C_{3}\right) \rtimes C_{2}\right.$}

Group $G_{4}$ has the following presentation:

$$
\begin{gathered}
G_{4}=\langle x, y, z, w, t| x^{2},[y, x],[z, x] z^{-1},[w, x] w^{-1},[t, x], y^{2},[z, y],[w, y],[t, y] t^{-1}, z^{3}, \\
\left.[w, z] t^{-1},[t, z], w^{3},[t, w], t^{3}\right\rangle .
\end{gathered}
$$

\begin{tabular}{|c|c|c|c|c|c|c|c|c|c|c|c|c|c|c|c|c|c|c|c|}
\hline r & $\begin{array}{l}e x \\
\end{array}$ & $y$ & & $w$ & $t x$ & $x y$ & $x t$ & $y z$ & $y w$ & $y t$ & $z w$ & $t^{2}$ & xyt & $x t^{2}$ & $y z w$ & $y t^{2}$ & $z^{2} w$ & $x y t^{2}$ & $y z^{2} w$ \\
\hline 7 & \begin{tabular}{l|l}
1 & 9
\end{tabular} & 1 & & 6 & 15 & 9 & 9 & 6 & 6 & 1 & 6 & 1 & 9 & 9 & 6 & 1 & 6 & 9 & 6 \\
\hline O & \begin{tabular}{l|l}
1 & 2 \\
\end{tabular} & 2 & & 3 & \begin{tabular}{l|l}
3 & 2 \\
\end{tabular} & 2 & 6 & 6 & 6 & 6 & 3 & 3 & 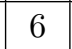 & 6 & 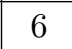 & 6 & 3 & 6 & 6 \\
\hline
\end{tabular}

Further, $G_{4}$ has 20 conjugacy classes as shown in the table below.

From above discussion, clearly the exponent of $G_{4}$ is 6 . Also $G_{4}^{\prime} \cong\left(C_{3} \times C_{3}\right) \rtimes C_{3}$ with $G_{4} / G_{4}^{\prime} \cong C_{2} \times C_{2}$. Next, we discuss the unit group of $\mathbb{F}_{q} G_{4}$ when $p>3$.

Theorem 3.4. The unit group of $\mathbb{F}_{q} G_{4}$, for $q=p^{k}, p>3$ where $\mathbb{F}_{q}$ is a finite field having $q=p^{k}$ elements is as follows:

1. for any $p$ and $k$ even or $p^{k} \equiv 1 \bmod 6$ with $k$ odd, we have

$$
U\left(\mathbb{F}_{q} G_{4}\right) \cong\left(\mathbb{F}_{q}^{*}\right)^{4} \oplus G L_{2}\left(\mathbb{F}_{q}\right)^{8} \oplus G L_{3}\left(\mathbb{F}_{q}\right)^{8} .
$$

2. for $p^{k} \equiv 5 \bmod 6$ with $k$ odd, we have

$$
U\left(\mathbb{F}_{q} G_{4}\right) \cong\left(\mathbb{F}_{q}^{*}\right)^{4} \oplus G L_{2}\left(\mathbb{F}_{q}\right)^{8} \oplus G L_{3}\left(\mathbb{F}_{q^{2}}\right)^{4}
$$

Proof. Since $\mathbb{F}_{q} G_{4}$ is semisimple, we have

$$
\mathbb{F}_{q} G_{4} \cong \mathbb{F}_{q} \oplus_{r=1}^{t-1} M_{n_{r}}\left(\mathbb{F}_{r}\right), \text { for some } t \in \mathbb{Z} \text {. }
$$

First assume that $k$ is even which means for any prime $p>3, p^{k} \equiv 1 \bmod 6$. This means $\left|S\left(\gamma_{g}\right)\right|=1$ for each $g \in G_{4}$. Hence, (17), Theorems 2.1 and 2.2 imply that

$$
\mathbb{F}_{q} G_{4} \cong \mathbb{F}_{q} \oplus_{r=1}^{19} M_{n_{r}}\left(\mathbb{F}_{q}\right)
$$

Using Theorem 2.5 with $G_{4}^{\prime} \cong\left(C_{3} \times C_{3}\right) \rtimes C_{3}$ in above to obtain

$$
\mathbb{F}_{q} G_{4} \cong \mathbb{F}_{q}^{4} \oplus_{r=1}^{16} M_{n_{r}}\left(\mathbb{F}_{q}\right) \text {, where } n_{r} \geq 2 \text { with } 104=\sum_{r=1}^{16} n_{r}^{2} .
$$

Above equation gives us the only possibility $\left(2^{8}, 3^{8}\right)$ for values of $n_{r}^{\prime} \mathrm{s}$ which means the required Wedderburn decomposition is

$$
\mathbb{F}_{q} G_{4} \cong \mathbb{F}_{q}^{4} \oplus M_{2}\left(\mathbb{F}_{q}\right)^{8} \oplus M_{3}\left(\mathbb{F}_{q}\right)^{8}
$$


Now we consider that $k$ is odd. We shall discuss this possibility in the following two cases: Case 1: $p^{k} \equiv 1 \bmod 6$. In this case, Wedderburn decomposition is given by (19).

Case 2. $p^{k} \equiv 5 \bmod 6$. In this case, we have

$$
S\left(\gamma_{t}\right)=\left\{\gamma_{t}, \gamma_{t^{2}}\right\}, S\left(\gamma_{x t}\right)=\left\{\gamma_{x t}, \gamma_{x t^{2}}\right\}, S\left(\gamma_{y t}\right)=\left\{\gamma_{y t}, \gamma_{y t^{2}}\right\}, S\left(\gamma_{x y t}\right)=\left\{\gamma_{x y t}, \gamma_{x y t^{2}}\right\},
$$

and $S\left(\gamma_{g}\right)=\left\{\gamma_{g}\right\}$ for all the remaining representatives $g$ of conjugacy classes. Hence, (17), Theorems 2.1 and 2.2 imply that

$$
\mathbb{F}_{q} G_{4} \cong \mathbb{F}_{q} \oplus_{r=1}^{11} M_{n_{r}}\left(\mathbb{F}_{q}\right) \oplus_{r=12}^{15} M_{n_{r}}\left(\mathbb{F}_{q^{2}}\right) .
$$

Above with Theorem 2.5 yields

$$
\mathbb{F}_{q} G_{4} \cong \mathbb{F}_{q}^{4} \oplus_{r=1}^{8} M_{n_{r}}\left(\mathbb{F}_{q}\right) \oplus_{r=9}^{12} M_{n_{r}}\left(\mathbb{F}_{q^{2}}\right), \text { where } n_{r} \geq 2 \text { with } 104=\sum_{r=1}^{8} n_{r}^{2}+2 \sum_{r=9}^{12} n_{r}^{2}
$$

\begin{tabular}{|c|c|c|c|c|c|c|c|c|c|c|}
\hline r $e e$ & $\begin{array}{lll}x & y \\
\end{array}$ & $y z$ & & $x y$ & $y 2$ & $y w$ & $z u$ & $y z w$ & $z^{2} w$ & $y z^{2} u$ \\
\hline \begin{tabular}{l|l}
$\mathrm{s}$ & 1
\end{tabular} & \begin{tabular}{l|l}
9 & 1 \\
\end{tabular} & 12 & 2 & 9 & 2 & 2 & 2 & 2 & 2 & 2 \\
\hline \begin{tabular}{l|l} 
o \\
\end{tabular} & & & & & 6 & & 3 & 6 & 3 & 6 \\
\hline
\end{tabular}

Further, consider the normal subgroup $H_{4}=\langle t\rangle$ of $G_{4}$ having order 3 with $K_{4}=G_{4} / H_{4} \cong C_{2} \times\left(\left(C_{3} \times\right.\right.$ $\left.C_{3}\right) \rtimes C_{2}$ ). It can be verified that $K_{4}$ has 12 conjugacy classes as shown in the table below.

It can be seen that for all the representatives $g$ of $K_{4},\left|S\left(\gamma_{g}\right)\right|=1$ which means by Theorems 2.1 and 2.2, we have

$$
\mathbb{F}_{q} K_{4} \cong \mathbb{F}_{q} \oplus_{r=1}^{11} M_{t_{r}}\left(\mathbb{F}_{q}\right), t_{r} \in \mathbb{Z} .
$$

Observe that $K_{4} / K_{4}^{\prime} \cong C_{2} \times C_{2}$. This with above and Theorem 2.5 imply that

$$
\mathbb{F}_{q} K_{4} \cong \mathbb{F}_{q}^{4} \oplus_{r=1}^{8} M_{t_{r}}\left(\mathbb{F}_{q}\right), \text { with } 32=\sum_{r=1}^{8} t_{r}^{2}, t_{r} \geq 2 .
$$

This gives us the only choice $\left(2^{8}\right)$ for values of $t_{r}^{\prime}$ s. Therefore, Theorem 2.6 and (20) yields

$$
\mathbb{F}_{q} G_{4} \cong \mathbb{F}_{q}^{4} \oplus M_{2}\left(\mathbb{F}_{q}\right)^{8} \oplus_{r=1}^{4} M_{n_{r}}\left(\mathbb{F}_{q^{2}}\right), n_{r} \geq 2 \text { with } 36=\sum_{r=1}^{4} n_{r}^{2}
$$

Above leaves us with the only choice $\left(3^{4}\right)$ for values of $n_{r}^{\prime}$ s which means the required Wedderburn decomposition is

$$
\mathbb{F}_{q} G_{4} \cong \mathbb{F}_{q}^{4} \oplus M_{2}\left(\mathbb{F}_{q}\right)^{8} \oplus M_{3}\left(\mathbb{F}_{q^{2}}\right)^{4}
$$

\section{Discussion}

We have characterized the unit groups of semisimple group algebras of 4 non-metabelian groups having order 108 and the results are verified using GAP. Clearly, the complexity in the calculation of Wedderburn decomposition upsurges with the increase in order of the group and we need to look into the Wedderburn decompositions of the quotient groups. The technique used for obtaining the Wedderburn decomposition works well provided the group has non-trivial normal subgroups of small order. 


\section{References}

[1] A. Bovdi, J. Kurdics, Lie properties of the group algebra and the nilpotency class of the group of units, J. Algebra 212 (1999) 28-64.

[2] V. Bovdi, M. Salim, On the unit group of a commutative group ring, Acta Sci. Math. (Szeged) 80 (2014) 433-445.

[3] R. A. Ferraz, Simple components of the center of FG/J(FG), Comm. Algebra 36 (2008) 3191-3199.

[4] B. Hurley, T. Hurley, Group ring cryptography, Int. J. Pure Appl. Math. 69 (2011) 67-86.

[5] P. Hurley, T. Hurley, Codes from zero-divisors and units in group rings, Int. J. Inf. Coding Theory 1 (2009) 57-87.

[6] G. Karpilovsky, The Jacobson radical of group algebras, Volume 135 Elsevier (1987).

[7] M. Khan, R. K. Sharma, J. Srivastava, The unit group of F $S_{4}$, Acta Math. Hungar. 118 (2008) 105-113.

[8] R. Lidl, H. Niederreiter, Introduction to finite fields and their applications, Cambridge university press (1994).

[9] S. Maheshwari, R. K. Sharma, The unit group of group algebra $F_{q} S L\left(2, Z_{3}\right)$, J. Algebra Comb. Discrete Appl. 3 (2016) 1-6.

[10] N. Makhijani, R. K. Sharma, J. Srivastava, A note on the structure of $F_{p^{k}} A_{5} / J\left(F_{p^{k}} A_{5}\right)$, Acta Sci. Math. (Szeged) 82 (2016) 29-43.

[11] C. P. Milies, S. K. Sehgal, An introduction to group rings, Springer Science \& Business Media (2002).

[12] G. Mittal, R. K. Sharma, On unit group of finite group algebras of non-metabelian groups up to order 72, Math Bohemica (2021)

[13] G. Pazderski, The orders to which only belong metabelian groups, Math. Nachr. 95 (1980) 7-16.

[14] S. Perlis, G. L. Walker, Abelian group algebras of finite order, Trans. Amer. Math. Soc. 68 (1950) $420-426$.

[15] R. K. Sharma, G. Mittal, On the unit group of a semisimple group algebra $\mathbb{F}_{q} S L\left(2, \mathbb{Z}_{5}\right)$, Math Bohemica (2021). 